identity mapping $i$ is semi-connected, by (7). Hence (b) implies (c). If (c) holds then $i$ is a homeomorphism. By Theorem 2, (X, $u$ ) is s.l.c.

\title{
BIBLIOGRAPHY
}

1. G. T. Whyburn, Analytic topology, Amer. Math. Soc. Colloq. Publ. Vol. 28, Amer. Math. Soc., Providence, R. I., 1942.

2. T. Tanaka, On the family of connected subsets and the topology of space., J. Math. Soc. Japan 7 (1955), 389-393.

3. W. J. Pervin and N. Levine, Connected mappings of Hausdorff space, Proc. Amer. Math. Soc. 11 (1960), 688-691.

UNIVERSITY OF CONNECTICUT AND UNIVERSITY OF OREGON

\section{A TECHNIQUE FOR CONSTRUCTING EXAMPLES}

\section{MARY ELLEN RUDIN}

The word space in this paper will refer to Hausdorff spaces.

I have recently been asked the following questions.

1 (by the topology class of R. H. Bing). Is there a regular, sequentially compact space in which some nested sequence of continua intersect in a disconnected set?

2 (by E. Michael). Is there a normal, sequentially compact but not compact, space having a separable, metric, locally compact, dense subset?

Examples showing that the answer to both questions is yes, modulo the continuum hypothesis, are easily constructed using a technique I have of ten used before. The technique, described in $\S I$, is perhaps more interesting than the particular examples which are given in \$II. §III gives a variation of the technique and raises some questions.

I. This technique is useful in the construction of pathological spaces having nice dense subsets.

Let $R$ be the wedge in the plane consisting of all points $(x, y)$ such that $0 \leqq x \leqq 1$ and $0 \leqq y \leqq x$; let $T=R-\{(0,0)\}$.

Let $F$ be the set of all continuous real valued functions whose domain is the set of all positive numbers less than or equal to 1 and whose graph lies in $T$.

There is a natural partial ordering of the terms of $F$ : if $f$ and $g$

Received by the editors December 16, 1964. 
belong to $F$, define $f<g$ if and only if there is a positive number $\epsilon$ such that, for all $x<\epsilon, f(x)<g(x)$.

Observation 1. If $A$ and $B$ are countable subsets of $F$ and every term of $A$ is <every term of $B$, then there is a term $f$ of $F$ such that every term of $A$ is $<f$ and $f$ is <every term of $B$.

Observation 1 assures us of many kinds of uncountable totally ordered by < subsets of $F$. And "the technique" is the replacement of the point $(0,0)$ of $R$ by some uncountable totally ordered by $<$ subset of $F$ which has whatever property is desired. The set $T$ preserves its simple plane topology and the subset of $F$ has the interval topology inducted by <; but the neighborhoods of terms of $F$ reach into $T$ by wedge shaped open sets of some $\epsilon$ length along the graphs of each term of $F$ in the neighborhood. One may also add some Dedekind completion to the subset of $F$ in the space. In either case the space is easy to visualize.

II. In order to give some special examples of the technique, let us define $a$ and $z$ as the terms of $F$ whose graphs are the intersection of $T$ and the lines $y=0$ and $y=x$, respectively.

Observation 2. If $A$ is a countable, totally ordered by $<$ subset of $F$ to which $a$ and $z$ belong, and $E$ is any subset of $T$ having $(0,0)$ in its closure, then there is a term $f$ of $F$ whose graph intersects $E$ in infinitely many points such that $(A \cup\{f\})$ is totally ordered by $<$.

Using Observation 2 and the obvious transfinite induction one arrives at Observation 3.

OBSERVATION 3. If the hypothesis of the continuum is true, there is a totally ordered by < subset $G$ of $F$ such that, if $E$ is any subset of $T$ having $(0,0)$ in its closure, then there is a term of $G$ whose graph intersects $E$ in infinitely many points.

Define $G$ as in Observation 3 ; clearly $G$ is a maximal totally ordered by $<$ subset of $F$ to which $a$ and $z$ belong. Define $Q$ as the set of all subsets $A$ of $G$ such that every term of $A$ is < every term of $(G-A)$ and there is no last term of $A$ or first of $(G-A)$.

Let $S=G \cup Q$. The ordering < of $G$ can be extended to $S$ : if $A$ and $B$ belong to $Q$, define $A<B$ if $A \subset B$, and, if $A$ belongs to $Q$ and $g$ to $G$, define $g<A$ if $g \in A$ and $A<g$ if $g \notin A$. The space resulting from using the induced topology defined by $<$ on $S$ is compact and connected.

For each positive number $\epsilon$ and each two terms $f<g$ of $F$, define $U_{\text {foe }}$ to be the subset $U$ of $(T \cup S)$ such that:

(1) $U \cap S$ is the set of all terms of $S$ between $f$ and $g$ in $<$, and 
(2) $U \cap T$ is the set of all points $(x, y)$ of $T$ such that $x<\epsilon$ and $f(x)<y<g(x)$.

Let $M$ be the set of all $U$ for which there is a positive number $\epsilon$ and two terms $f<g$ of $F$ such that either

(1) $U=U_{\text {foe }}$, or

(2) $f=a$ and $U=U_{a g \epsilon} \cup\{a\} \cup\{(x, 0) \mid x<\epsilon\}$, or

(3) $g=z$ and $U=U_{f z \epsilon} \cup\{z\} \cup\{(x, x) \mid x<\epsilon\}$.

Let $N$ be the set of all intersections of $T$ with open sets in the plane.

EXAMPLE 0. Let $N \cup M$ be a neighborhood basis for the space $\Sigma_{0}$ whose points are the elements of $S \cup T$.

The space $\Sigma_{0}$ is normal, connected, and compact, and, even though the space is far from metric, it seems almost too nice to be useful. Modifications however will answer the questions mentioned at the beginning. However we need the following.

OBSERVATION 4. If the hypothesis of the continuum is true, there is a term $X$ of $Q$ and, for each countable ordinal $\alpha$, terms $X_{\alpha}$ and $X_{\alpha}^{\prime}$ of $Q$ such that:

(1) $X_{\alpha}$ is a proper subset of $X_{\alpha+1}$ and $X_{\alpha+1}^{\prime}$ is a proper subset of $X_{\alpha}^{\prime}$,

(2) $X=\cup_{\text {all } \alpha} X_{\alpha}=\bigcap_{\text {all } \alpha} X_{\alpha}^{\prime}$, and

(3) if $\alpha$ is a limit ordinal, then $X_{\alpha}=\cup_{\beta<\alpha} X_{\beta}$ and $X_{\alpha}^{\prime}=X_{\beta<\alpha} \cap X_{\beta}^{\prime}$.

The sets $x$ and $x^{\prime}$ of all $X_{\alpha}$ and $X_{\alpha}^{\prime}$, respectively, will be used in example 2 ; let $S$ and $\mathcal{S}^{\prime}$ denote the set of all terms of $S$ which precede and follow $X$, respectively, in $<$.

EXAMPLE 1. Let $\Sigma_{1}=\Sigma_{0}-\{X\}$.

Then $\Sigma_{1}$ is regular, connected, sequentially compact, and locally compact, although no longer compact or normal since there are no disjoint open sets containing the mutually separated continua $S$ and $\mathcal{S}^{\prime}$ of $\Sigma_{1}$. And, since $\mathcal{S} \cup \mathcal{S}^{\prime}$ is the intersection of the nested sequence of continua $\left\{s \cup \mathcal{S}^{\prime} \cup C_{n}\right\}$ where, for each positive integer $n, C_{n}$ is the intersection of $T$ and the circle plus its interior in the plane having radius $1 / n$ and center $(0,0), \Sigma_{1}$ answers question 1 . Observe that no normal, sequentially compact topological space has a nested sequence of continua whose intersection is not connected.

Let $M^{\prime}$ be the set of all $U$ such that either

(1) $U$ belongs to $M$ and $\left(U \cap\left(x \cup X^{\prime}\right)\right)=0$, or

(2) there are terms $V$ and $V^{\prime}$ of $M$ such that $V \cap x^{\prime}=0$ and $V^{\prime} \cap x=0$ and, for each countable ordinal $\alpha, X_{\alpha}$ belongs to $V$ if and only if $X_{\alpha}^{\prime}$ belongs to $V^{\prime}$, and $U=\left(V \cup V^{\prime}\right)-\{X\}$.

EXAMPLE 2. Let $N \cup M^{\prime}$ be a neighborhood basis for the space $\Sigma_{2}$ whose points are the elements of $T \cup(S-(\{X\} \cup x))$.

The only difference between the spaces $\Sigma_{1}$ and $\Sigma_{2}$ is that, for each 
$\alpha$, the points $X_{\alpha}$ and $X_{\alpha}^{\prime}$ are identified in $\Sigma_{2}$ and hence there are no two noncompact disjoint closed subsets of $\Sigma_{2}$. Therefore, $\Sigma_{2}$ is normal, sequentially compact, and locally compact, but not compact, and the simple plane triangle without one vertex, $T$, is dense in $\Sigma_{2}$; which answers question 2 .

The virtue of this technique as illustrated by the examples is that while the properties desired and the spaces described sound messy, the spaces are easily seen geometrically.

III. One pertinent fact is that we use the continuity of the terms of $F$ in precisely two ways:

(1) the number of elements of $F$ is power of the continuum, and

(2) each $U_{f o \epsilon}$ is an open subset of $T$ and our spaces are thus regular.

From this one can see that we can vary our technique by taking our nice dense set to be a countable discrete set rather than a connected planar set. For instance, define $T$ as the set of all points $(1 / n, 1 / m)$ in the plane where $n$ and $m$ are integers and $n \leqq m \leqq 2 n$; and define $F$ as the set of all real valued functions whose domain is the set of all reciprocals of positive integers and whose graphs lie in $T$. The resulting space $\Sigma_{2}$ would be normal, sequentially and locally compact, but not compact, and the set $T$ is countable, discrete, and dense in $\Sigma_{2}$.

I would also like to raise two questions concerning maximal totally ordered by < sets similar to $G$.

Observe that, if the hypothesis of the continuum is true, any two maximal totally ordered by < subsets of $F$ are order isomorphic. However I do not know the answer to the following:

If the hypothesis of the continuum is not true, are there maximal totally ordered by < subsets of $F$ of different cardinality?

Similarly, suppose one leaves the word "continuous" out of the definition of $F$; then the cardinality of $F$ is greater than that of the continuum. One can still make Observations 1 and 2, but I cannot say much more. I would even like to know the answer to the following:

If the word "continuous" is deleted from the definition of $F$, is there a totally ordered by $<$ subset of $F$ of cardinality greater than that of the continuum?

UNIVERSITY OF WISCONSIN 